\title{
Numerical Simulation of Molten Steel Flow under a Magnetic Field with Argon Gas Bubbling in a Continuous Casting Mold
}

\author{
Noriko KUBO, Toshio ISHII, Jun KUBOTA ${ }^{1)}$ and Toru IKAGAWA ${ }^{11}$ \\ JFE R\&D Corp., 1-1 Minamiwatarida-cho, Kawasaki-ku, Kawasaki 210-0855 Japan. \\ Fukuyama, Hiroshima 721-8510 Japan.
}

1) JFE Steel Corp., 1 Kokan-cho,

(Received on July 9, 2003; accepted in final form on October 24, 2003)

\begin{abstract}
In a continuous casting process, it is essential to prevent the surface defects which are caused by the entrapments of both mold powder and argon gas bubbles. It is well known through experiments that the decrease in the molten steel flow velocity just under the free surface is one of the most effective methods for the prevention of mold powder entrapments. For this purpose, we have already employed the electromag-

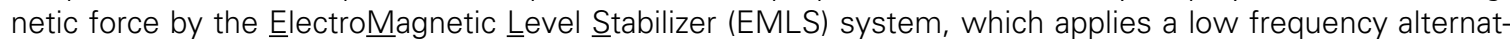
ing magnetic field that moves from the narrow face of the mold to the mold center below the nozzle exits. In this study, the molten steel flow in a mold was numerically analyzed to optimize the conditions for the prevention of the deteriorating phenomena: optimization of electromagnetic force with argon gas bubbling.

Simulation results indicate that argon gas bubbles ascend near the nozzle due to their buoyancy, and ascending argon bubbles induce the upstream of the molten steel. Due to the electromagnetic force, the molten steel is forced to flow toward the magnetic field traveling direction in the region where the magnetic field is imposed. Consequently, the molten steel flows toward the mold center near the free surface with a smaller imposed magnetic field, and it flows toward the narrow face with a larger imposed magnetic filed. A suitable imposed magnetic filed with argon gas bubbling can be chosen to minimize the flow velocity and also the amount of mold powder entrapments.
\end{abstract}

KEY WORDS: continuous casting; mold; quality control; simulation; molten steel flow; argon gas; electromagnetic force.

\section{Introduction}

High quality steel products are strongly demanded. As the continuous casting process is closely related to the quality of steel products, it is essential to supply clean molten steel to the mold and to prevent the entrapment of impurities. In an operation, the mold powder is used to keep high temperature and adequate lubrication between the mold and the solidification shell. Argon gas is supplied to the mold to prevent the immersion nozzle from clogging. However, both the mold powder and argon gas are potentially causes of the surface defects; in certain conditions, these are easily entrapped into the solidification shell of the steel.

As it is well known through experiments, when the molten steel flow pattern in the mold is not symmetric on the right and left side of the immersion nozzle, the molten steel sometimes swirls at the free surface and inclusions such as the mold powder and argon gas are caught in the vortex. ${ }^{1-3)}$ In addition, the more the casting speed is increased, the more inclusions are entrapped. Particularly, the horizontal molten steel flow velocity just under the free surface, referred to as "surface velocity", affects the frequency and the amount of entrapments. ${ }^{3-11)}$ R. Nishimachi et al. ${ }^{9 \text { ) }}$ indicates that the relationship between the surface velocity and the surface defects in cold rolled coils of practical casting shown in Fig. 1. The plus sign of the horizontal axis stands for the velocity in the direction toward the nozzle and vice visa. When the surface velocity is close to zero, the index of the surface defects is kept very low. It means that the frequency of the defects strongly depends on the surface velocity. Thus, to decrease the surface velocity is one of the most effective methods to reduce the defects.

In order to reduce the surface velocity, we have already employed the electromagnetic force by the EMLS system, ${ }^{12)}$ which applies a low frequency alternating magnetic field that moves from the narrow face of the mold to the

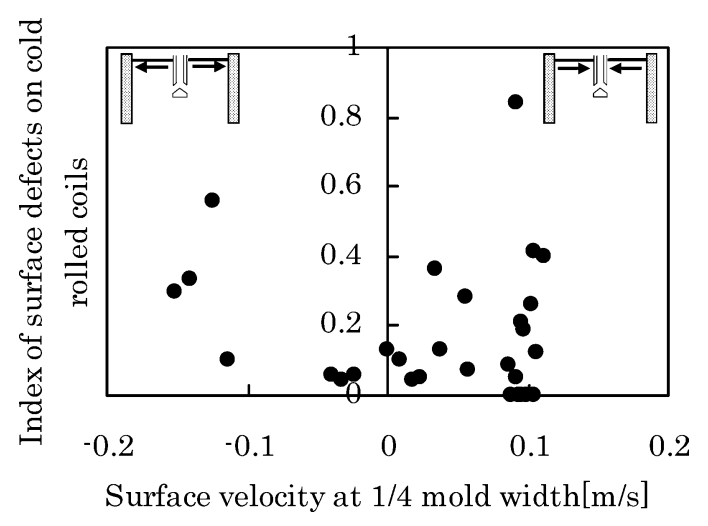

Fig. 1. Relationship between surface velocity and surface defects in cold rolled coils. ${ }^{9)}$ 


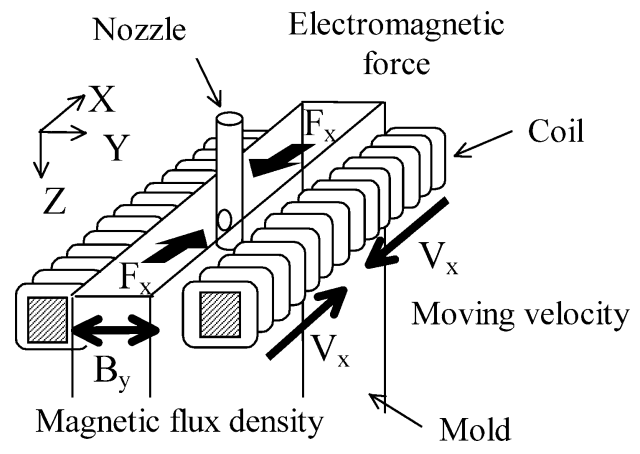

Fig. 2. Schematic diagram of EMLS system.

mold center below the nozzle exit. The schematic diagram of the EMLS system is shown in Fig. 2. The electromagnetic force applying the magnetic field can be estimated. The EMLS installation is composed of some coils side by side. An alternating electric current flows in one coil with a certain different phase to that of another coil. Therefore, the magnetic pole moves in a certain direction with time. The moving velocity of the magnetic pole $V_{x}$ is calculated with the pole pitch (distance from $\mathrm{N}$ pole to $\mathrm{S}$ pole) $\tau$ and the frequency $f$ shown in Eq. (1).

$$
V_{x}=2 \tau f
$$

An electric current $J_{z}$ is induced by the Lorentz law shown in Eq. (2).

$$
J_{z}=\sigma V_{x} B_{y}
$$

where, $\sigma$ is the electrical conductivity of the material and $B_{y}$ is the magnetic flux density. Consequently, the electromagnetic force is estimated in Eq. (3).

$$
\begin{aligned}
F_{x} & =J_{z} B_{y} \\
& =2 \tau \sigma f B_{y}^{2}
\end{aligned}
$$

This equation shows that the main direction of the electromagnetic force is same as the direction in which the magnetic pole moves. In the EMLS system, as the magnetic pole moves from the narrow face of the mold to the mold center, the molten steel flow from the nozzle will be restrained by the electromagnetic force.

Some kinds of magnetic field imposing systems such as a static magnetic field or a traveling magnetic field have been practically used. Therefore, there are a lot of studies that deal with the molten steel flow in a mold under a magnetic field. ${ }^{13-20)}$ It is reported that argon gas is able to change the molten steel flow pattern in a mold due to the difference of the specific gravity ${ }^{21-27)}$ and causes a fluctuation at the free surface. Because of that, some studies treating the electromagnetic force take argon gas into consideration. ${ }^{28,29)}$ As the braking effect of the EMLS system with argon gas bubbling has not been discussed so far, this study focuses on it. The flow of the molten steel in a mold is numerically analyzed to optimize the electromagnetic force for the prevention of the deteriorating phenomena. In the calculation, commercial package software for computational fluid dynamics is used.

This study includes the investigation of the molten steel flow velocity and argon gas floating distribution in a practical continuous casting mold. To confirm the accuracy of the calculation, the computed results are drawn into comparison with the observation in the production machine.

\section{Calculation Method and Conditions}

\subsection{Calculation Method}

The molten steel is assumed to be incompressible and isothermal. The mass conservation equation and the momentum conservation equation for the molten steel flow are written in Eqs. (4) and (5).

$$
\begin{aligned}
& \frac{\partial u_{i}}{\partial x_{i}}=0 \\
& \frac{\partial}{\partial t}\left(\rho u_{i}\right)+\frac{\partial}{\partial x_{j}}\left(\rho u_{i} u_{j}\right) \\
& =-\frac{\partial p}{\partial x_{i}}+\frac{\partial}{\partial x_{j}} \mu_{e}\left(\frac{\partial u_{i}}{\partial x_{j}}+\frac{\partial u_{j}}{\partial x_{i}}\right)+\rho g_{i}+M_{i}+F_{i}
\end{aligned}
$$

where, $u$ is the flow velocity, $\rho$ is the specific density, $p$ is the pressure, $\mu_{e}$ is the effective viscosity, $g$ is the gravitational acceleration, $M$ is the momentum sink from the argon gas bubble to the molten steel and $F$ is the Lorentz Force.

The Reynolds number, $R_{e}$ estimated based on the dimensions of the immersion nozzle exit, is the order of $10^{5}$, which makes it necessary to take the effects of turbulence into account. The two-equation $k-\varepsilon$ model is adopted as a turbulence model:

$$
\begin{aligned}
& \mu_{e}=\mu+\mu_{t} \\
& \mu_{t}=C_{\mu} \rho \frac{k^{2}}{\varepsilon} \\
& \rho u_{i} \frac{\partial k}{\partial x_{i}}=\frac{\partial}{\partial x_{i}}\left(\frac{\mu_{t}}{\sigma_{k}} \frac{\partial k}{\partial x_{i}}\right)+G_{k}-\rho \varepsilon \\
& \rho u_{i} \frac{\partial \varepsilon}{\partial x_{i}}=\frac{\partial}{\partial x_{i}}\left(\frac{\mu_{t}}{\sigma_{\varepsilon}} \frac{\partial \varepsilon}{\partial x_{i}}\right)+C_{1 \varepsilon} \frac{\varepsilon}{k} G_{k}-C_{2 \varepsilon} \rho \frac{\varepsilon^{2}}{k} .
\end{aligned}
$$

where,

$$
\begin{aligned}
& G_{k}=\mu_{t} \frac{\partial u_{i}}{\partial x_{j}}\left(\frac{\partial u_{i}}{\partial x_{j}}+\frac{\partial u_{j}}{\partial x_{i}}\right) \\
& C_{1 \varepsilon}=1.44, C_{2 \varepsilon}=1.92, C_{\mu}=0.09, \sigma_{k}=1.0, \sigma_{e}=1.3
\end{aligned}
$$

$\mu$ is the molecular viscosity, $\mu_{t}$ is the turbulent viscosity, $k$ is the turbulent kinetic energy and $\varepsilon$ is the dissipation of turbulent kinetic energy. These basic equations are solved by the means of a 3-dimensional finite volume method. The boundary wall in the mold is assumed to be hydro-dynamically non-slip. A widely used wall function that was proposed by Launder and Spalding ${ }^{30)}$ is adopted. The boundary wall at the free surface is assumed to be slip because the mold powder on the meniscus is well melted.

As for argon gas, it is assumed to be incompressible and isothermal. The bubble shape is assumed to be spherical. The change in bubble size is not considered, so the effect of coalescence and breakup is neglected. Discrete Phase Model $^{31)}$ is used to consider the argon gas behavior for the reason of a light computational load ${ }^{32)}$ even when the parti- 
cle size distribution is set. The momentum equation for each bubble by the Lagrangian methods is written in Eq. (12).

$$
\begin{aligned}
& \frac{D u_{g}}{D t}=-K\left(\frac{u_{g}-u}{\rho_{g}}\right)+g\left(\frac{\rho-\rho_{g}}{\rho_{g}}\right) \\
& +\frac{1}{2} \frac{\rho}{\rho_{g}}\left(\frac{D u}{D t}-\frac{D u_{g}}{D t}\right)+\frac{\rho}{\rho_{g}} \frac{D u}{D t} \\
& K=\frac{3}{4} \rho C_{d} \frac{\left|u_{g}-u\right|}{d_{g}}, \quad C_{d}=\frac{24}{R_{e}}\left(1+0.15 R_{e}^{0.687}\right) \text {, } \\
& R_{e}=\frac{d_{g}\left|u_{g}-u\right| \rho}{\mu}
\end{aligned}
$$

where, $u_{g}$ and $\rho_{g}$ are the velocity and density of argon gas bubble respectively. $K$ is the interaction coefficient. $K$ is a function of the argon gas bubble diameter $d_{g}$, and the drag coefficient $C_{d}$ is a function of the Reynolds number $R_{e}$. Practically, each bubble trajectory is not tracked but some representative bubble trajectories are solved. The mass flow rate corresponding to the each representative bubbles is set.

Computing the argon gas trajectories, the momentum change of all the bubbles which pass through the control volume $\Delta V$ is summed and the quantity per unit volume is incorporated in the flow calculation as a momentum sink $M$.

$$
M=\frac{1}{\Delta V} \sum_{l=1}^{N} \int_{0}^{t_{r, l}} \frac{18 \mu}{\rho_{g} d_{g, l}^{2}} \frac{C_{d, l} R_{e, l}}{24}\left(u_{g, l}-u\right) m_{p, l} \mathrm{~d} t \ldots
$$

where, $m_{p}$ is the mass flow rate of the representative argon gas bubble, $N$ is the number of the representative bubbles which pass through the control volume and $t_{r}$ is the residence time in the control volume. This two-way coupling is accomplished by alternating the argon gas bubble calculation with the molten steel flow calculation.

When Eq. (12) is solved, we use the stochastic particle tracking method ${ }^{33)}$ in order to consider the turbulence dispersion of particles. As the molten steel flow velocity $u$, the fluctuating flow velocity $u^{\prime}$ is added to the mean flow velocity $\bar{u}$ in order to include the effect of turbulence dispersion of argon gas:

$$
u=\bar{u}+u^{\prime}
$$

The fluctuating flow velocity $u^{\prime}$ is expressed with a normally distributed random number $\zeta$ (namely, zero-mean, unitvariance) and the kinetic energy of turbulence $k$. The twoequation $k-\varepsilon$ model for isotropic turbulence is assumed here.

$$
u^{\prime}=\zeta \sqrt{\frac{2}{3} k}
$$

As for electromagnetic analysis, ${ }^{34,35)}$ we assume that the value of magnetic field at the boundary is given. The magnetic field inside the mold is obtained by solving Eq. (17).

$$
\frac{\partial B}{\partial t}=\nabla \times(u \times B)+\frac{1}{\mu_{m} \sigma} \nabla^{2} B
$$

where, $B$ is the magnetic flux density. $\mu_{m}$ is the magnetic permeability, which is assumed to be isotropic and constant $\left(1.257 \times 10^{6}[\mathrm{H} / \mathrm{m}]\right) . \sigma$ is the volume average of the molten steel electrical conductivity $\left(7.14 \times 10^{5}[1 / \Omega \mathrm{m}]\right)$ and argon gas electrical conductivity $(\approx 0[1 / \Omega \mathrm{m}])$.

$$
\sigma=\sum_{i} \sigma_{i} v_{i}
$$

When we obtain the distribution of magnetic fields inside the mold, we can calculate the induced current $J$ by the Eq. (19).

$$
J=\frac{1}{\mu_{m}}(\nabla \times B)
$$

Then, the Lorentz Force is obtained from the Eq. (20)

$$
F=J \times B
$$

This Lorentz Force is summed to the external force term of the flow momentum equation (see Eq. (5)).

\subsection{Calculation Conditions}

The calculation conditions are listed in Table 1. The calculated mold cavity is $1600 \times 200 \mathrm{~mm}$. The immersion nozzle is located at the center of the mold and has two rectangular exit ports. The port size is $100 \times 80 \mathrm{~mm}$. The upper edge of the nozzle exit port is $150 \mathrm{~mm}$ below the free surface. The molten steel flows through the immersion nozzle into the mold and exits at the bottom of it. The center of the EMLS device is located at $350 \mathrm{~mm}$ from the free surface, where it is just below the nozzle exit. The EMLS device generates a $1 \mathrm{~Hz}$-alternating magnetic field that moves from the narrow face of the mold to the mold center. One example of the magnetic flux density distribution along the mold width at the mold center-thickness and the coil center-depth is shown in Fig. 3. It can be seen that the maximum peak moves toward the mold center with time. Figure 4 shows the time average profile of the magnetic flux density distri-

Table 1. Calculation conditions.

\begin{tabular}{|l|c|}
\hline Mold size [mm] & $1600 \times 200$ \\
\hline Throughput [ton/min] & 5 \\
\hline Casting steed [m/min] & 2.2 \\
\hline Magnetic flux density B[Tesla] & $0 \sim 0.12$ \\
\hline Ar gas flow rate* Q[L/min] & 9 \\
\hline Ar gas volume fraction [\%] & 7.6 \\
\hline
\end{tabular}

* at standard condition

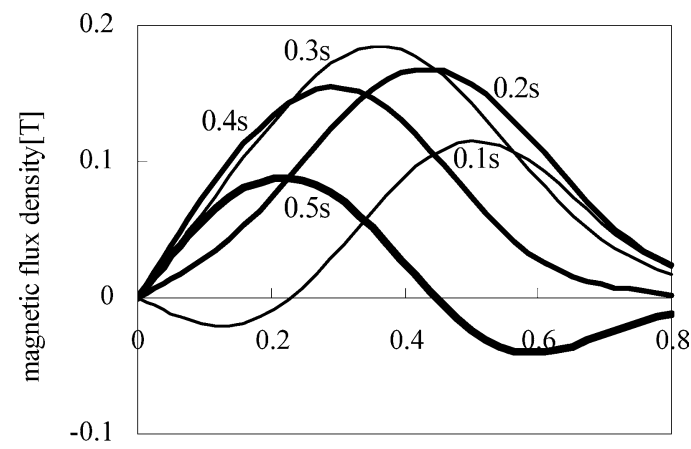

Horizontal position from the mold center[m]

Fig. 3. Imposed magnetic flux density distribution. 


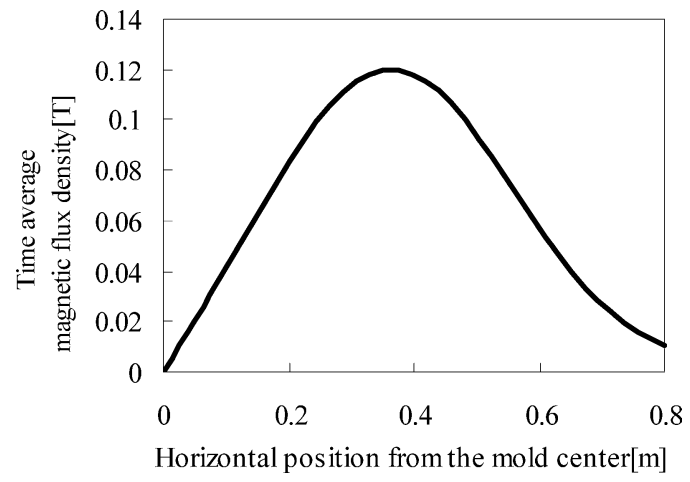

Fig. 4. Time average imposed magnetic flux density distribution.

butions. The magnetic flux density in Table 1 means the maximum value in the time average imposed magnetic field distribution for convenience' sake. We computed under the following conditions: 1) without both argon gas and a magnetic field, 2) with argon gas and without a magnetic field, 3) without argon gas and with a magnetic field, and 4) with both argon gas and a magnetic field. Calculations are performed for several different intensity of the imposed magnetic flux density. At first, steady state calculations are performed without imposing a magnetic field. Then, time-dependent calculations are carried out with imposing a magnetic field. The time step of the calculation is $0.1 \mathrm{~s}$. An imposed magnetic filed is interpolated at the same intervals and Eqs. (17)-(20) are solved every time. The Lorentz Force from the Eq. (20) is incorporated in a flow calculation as an external force. The calculation is carried on and when the flow becomes stable, the result is evaluated as a quasi-steady state.

Argon gas volume is assumed to inflate by 6 times in the molten steel due to thermal expansion. ${ }^{36}$ The argon gas volume fraction is defined as the ratio of argon gas flow rate to throughput of the molten steel. There is no general formula to express the bubble size distribution in the mold. However, there are some studies discussing the experimental bubble size distribution. For example, K. Takatani et $a l^{24)}$ investigated the bubble size in the low melting point alloy mold model. It is said that the mode diameter of the bubbles is around $1 \mathrm{~mm}$ and the volume of bubbles with a diameter over $5 \mathrm{~mm}$ is small. T. Toh et al. ${ }^{28)}$ investigated the bubble size in the mercury mold model. It is shown that the volume of very fine bubbles is less than $1 \%$ of the total argon gas volume. The bubbles with a diameter over $10 \mathrm{~mm}$ rarely have any effect on the molten steel flow, because they normally do not occur and just burst out intermittently. From these studies, it is considered that several $\mathrm{mm}$ in diameter bubbles play the important part in the formation of the molten steel flow pattern. In this size range, as the bubble size is larger, the number of bubbles and the flow rate of bubbles are smaller. In this paper, the Rosin-Rammler size distribution $^{31)}$ expressed in Eq. (21) is assumed as a simple function to fit the character as mentioned above. This function is widely used for the particle size distribution.

$$
M_{D}=\exp \left\{-(D / \bar{D})^{n}\right\}
$$

where, $D$ is the bubble size, $M_{D}$ is the mass fraction with the diameter greater than $D . \bar{D}$ is the representative bubble diameter when $M_{D}=1 / e . n$ is the spread parameter. The

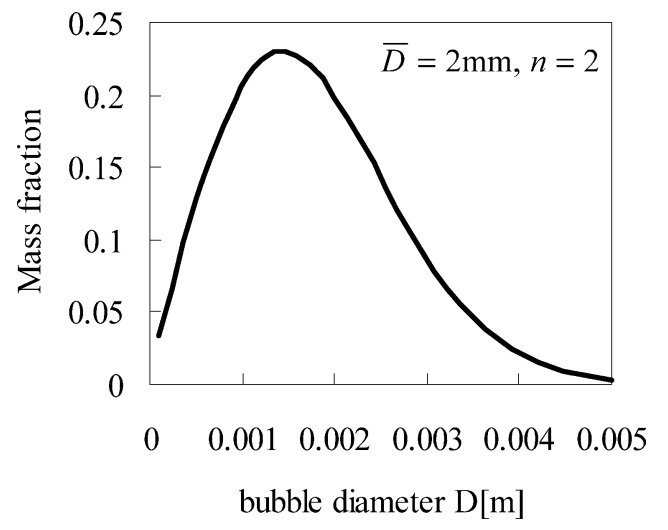

Fig. 5. Bubble size distribution.

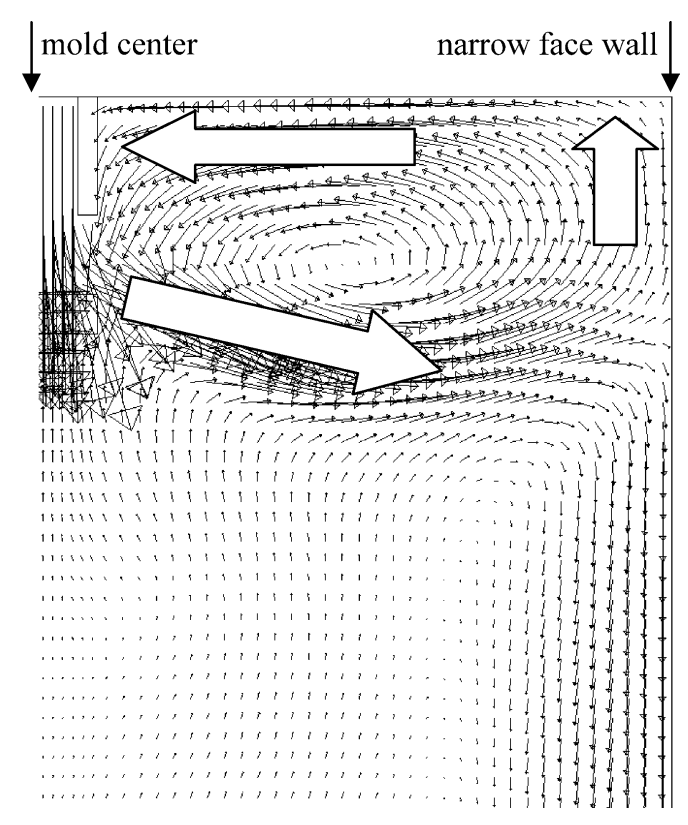

Fig. 6. Molten steel flow pattern $(Q=0 \mathrm{~L} / \mathrm{min}, B=0 \mathrm{~T})$.

minimum bubble diameter is set at $100 \mu \mathrm{m}$, the maximum bubble diameter is $5 \mathrm{~mm}$, the representative bubble diameter is $2 \mathrm{~mm}$ and the spread parameter is 2 . The adopted bubble size distribution is shown in Fig. 5. It should be noticed that the mode diameter is smaller than the representative bubble diameter, $2 \mathrm{~mm}$.

\section{Results}

\subsection{Calculated Molten Steel and Argon Gas Flow Pat- tern}

As a reference, a simple molten steel flow, when argon gas is not injected and a magnetic field is not applied, was calculated. The molten steel flow velocity vectors at the center-thickness are shown in Fig. 6. The picture is largescaled in the upper half and right side of the whole calculation region. In this case, supplied molten steel flows from the exit of the immersion nozzle to the narrow face of the mold, and separated at the mold wall. After the separation, some flows upwards and some flows downwards. Under a normal situation, argon gas is used for the prevention of the nozzle clogging. When argon gas is employed to the system, as shown in Fig. 7, the molten steel flows upward due to the floating argon gas bubbles close to the nozzle. 


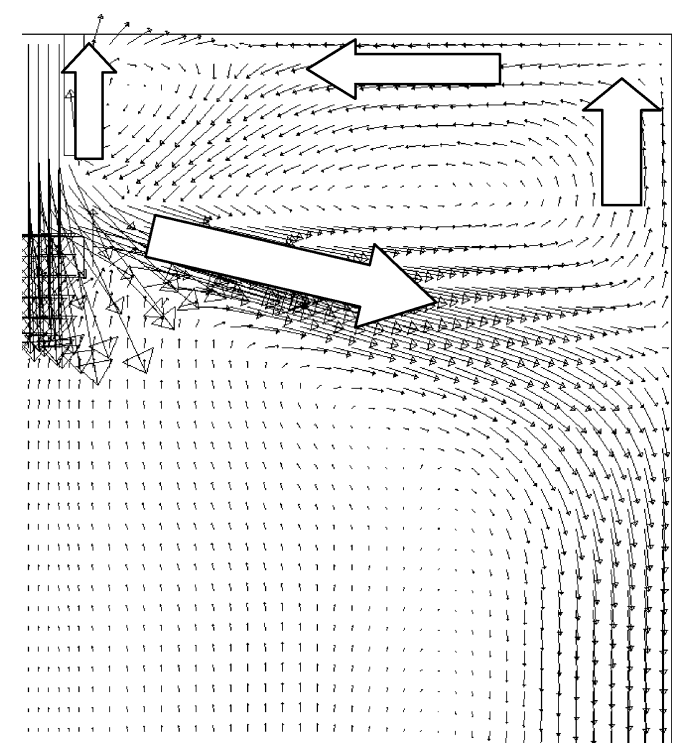

Fig. 7. Molten steel flow pattern $(Q=9 \mathrm{~L} / \mathrm{min}, B=0 \mathrm{~T})$.

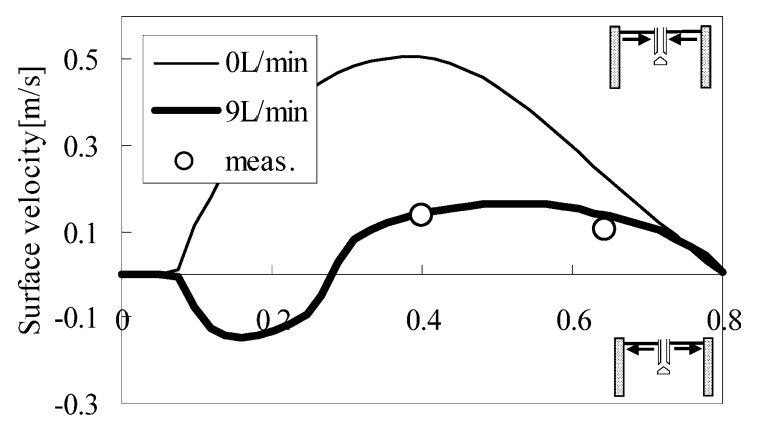

Horizontal position from the mold center[m]

Fig. 8. Surface velocity profiles at the center-thickness $(B=0 \mathrm{~T})$.

Surface velocity profiles for both the simple molten steel case and the argon gas bubbling case are plotted with lines in Fig. 8. From this figure, it can be said that argon gas is able to reduce the surface velocity, because argon gas dispersed the molten steel flow jet around the nozzle. The measured data under the argon gas bubbling is also plotted in Fig. 8. Measurement method is described for details in subsection 3.2.1. From the Fig. 8, calculated data and measured data are in good agreement with each other and it can be said that the selected bubble size distribution is reasonable at least in this calculation.

As a second stage, the molten steel flow was computed under a magnetic field without argon gas bubbling. The molten steel flow velocity vectors at the center-thickness are shown in Fig. 9. When the 0.072 Tesla magnetic flux density is applied, the molten steel is forced to flow toward the mold center just below the nozzle where the magnetic field is affected. As the molten steel flow jet from the nozzle is strong enough and breaks against the mold wall, the molten steel still flows from the narrow face of the mold to the nozzle near the free surface. Under the magnetic flux density of 0.096 Tesla, the molten steel flow jet from the nozzle is bent downward due to the electromagnetic force and does not reach the mold wall. Therefore, just near the free surface, the molten steel flow is weakened and flows toward the nozzle at the narrow face side and toward the (a) $\mathrm{B}=0.072 \mathrm{~T}$

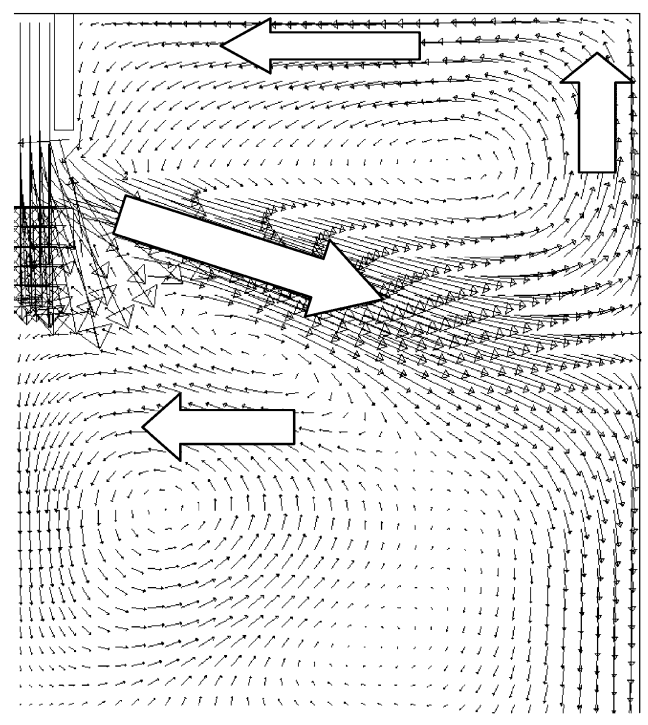

(b) $\mathrm{B}=0.096 \mathrm{~T}$

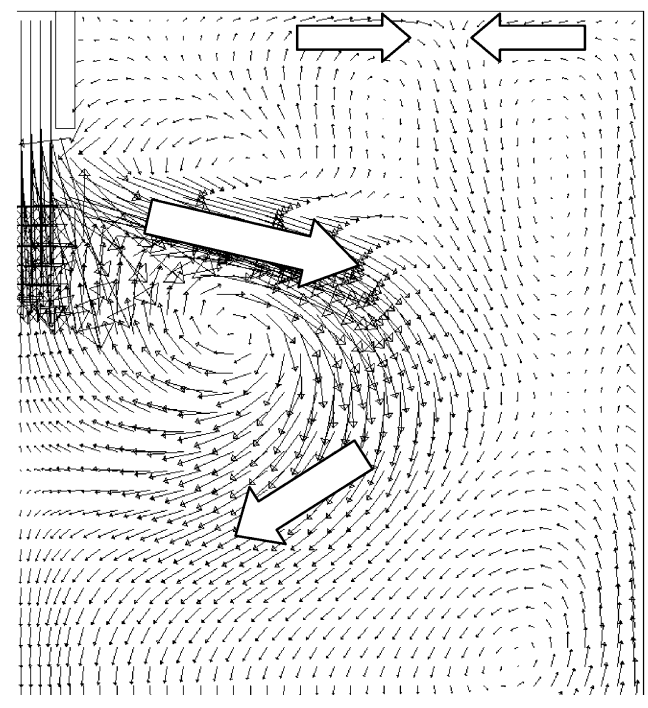

Fig. 9. Molten steel flow pattern $(Q=0 \mathrm{~L} / \mathrm{min})$. (a) $0.072 \mathrm{~T}$, (b) $0.096 \mathrm{~T}$.

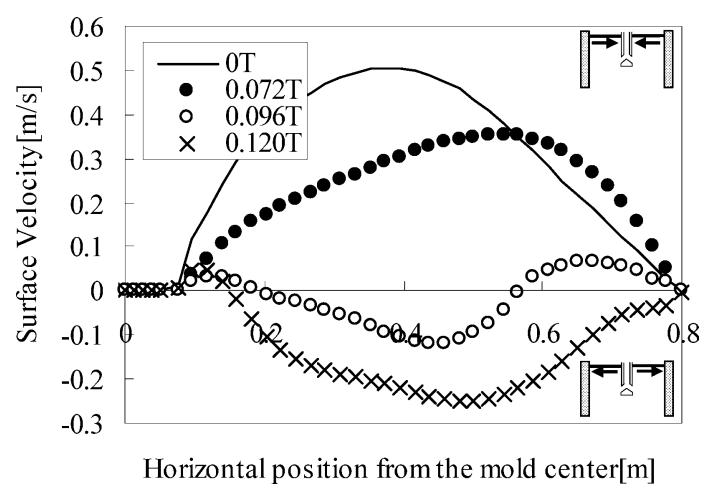

Fig. 10. Surface velocity profiles at the center-thickness $(Q=$ $0 \mathrm{~L} / \mathrm{min})$.

narrow face at the nozzle side. The surface velocity profiles for several different imposed magnetic flux densities are plotted in Fig. 10. The surface velocity is reduced with an increase in the intensity of the magnetic flux density, and 
(a) $0.072 \mathrm{~T}$

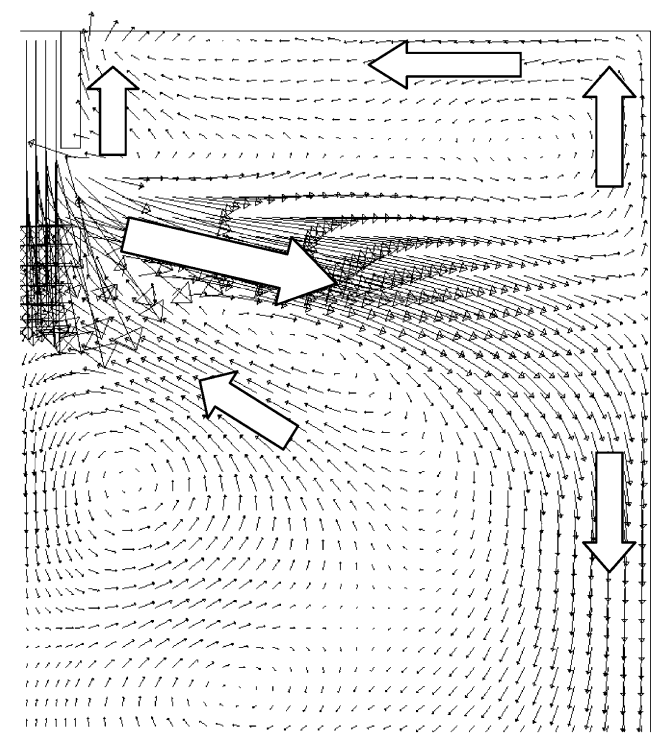

(b) $0.096 \mathrm{~T}$

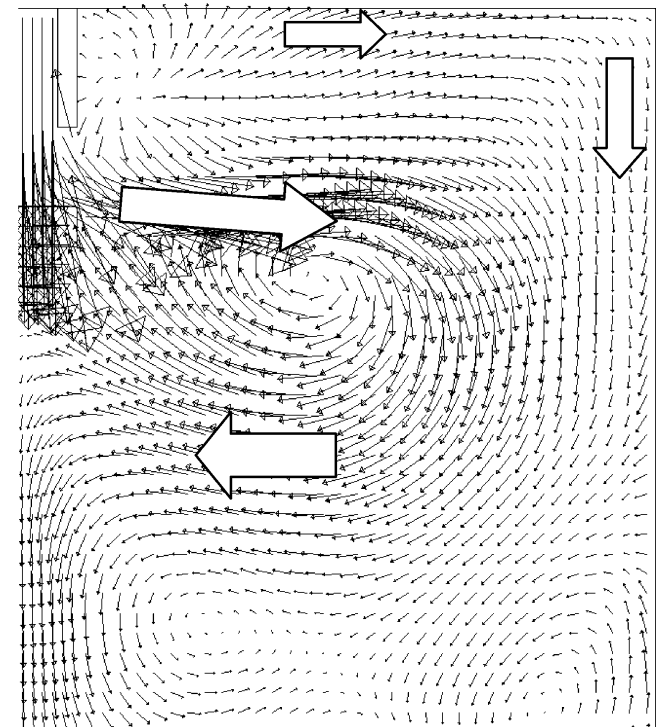

Fig. 11. Molten steel flow pattern $(Q=9 \mathrm{~L} / \mathrm{min})$. (a) $0.072 \mathrm{~T}$, (b) $0.096 \mathrm{~T}$. the surface flow direction is completely reversed at 0.120 Tesla when compared to no magnetic field case.

Next, the effect of both argon gas and a magnetic field was studied. The molten steel flow velocity vectors at the center-thickness are shown in Fig. 11. Although the floating argon gas lifts up the molten steel flow jet near the nozzle, the main jet reaches the narrow face of the mold wall under the 0.072 Tesla. As the jet separates into two ways at the mold wall, the molten steel near the free surface flows from the narrow face of the mold to the nozzle. When 0.096 Tesla is imposed, the molten steel is pushed toward the mold center in the magnetic field imposed area and the jet from the nozzle joins this induced flow. The molten steel near the free surface accompanies the jet from the nozzle. Compared to the no argon gas bubbling case, the molten steel near the free surface flows toward the narrow face with a smaller magnetic field. This is because argon gas makes the upstream of the molten steel near the nozzle and supports the flow toward the narrow face near the free surface. The surface velocity profiles for several different imposed magnetic flux densities are plotted in Fig. 12. The surface velocity is reduced with an increase in the intensity of the magnetic flux density, and the reversed surface flow region is rapidly increased because floating argon gas helps the molten steel flow toward the narrow face. The flow near the free surface is calmly stabilized around the 0.077 Tesla.

Argon gas trajectories are shown in Fig. 13. When the magnetic field is not applied, larger argon gas bubbles as-

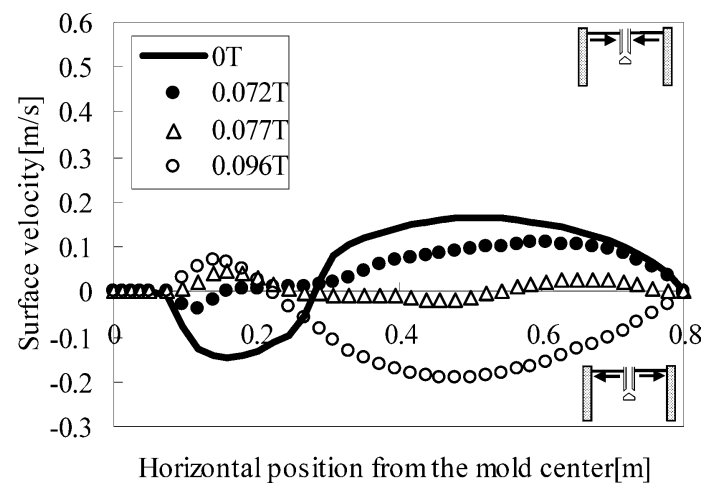

Fig. 12. Surface velocity profiles at the center-thickness $(Q=$ $9 \mathrm{~L} / \mathrm{min})$. (a) $0 \mathrm{~T}$

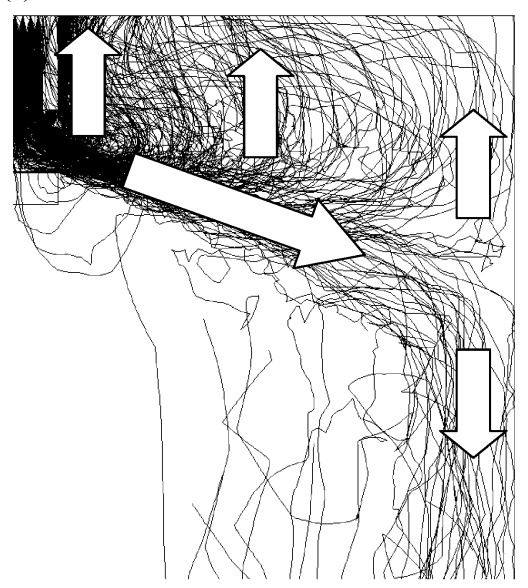

(b) $0.072 \mathrm{~T}$

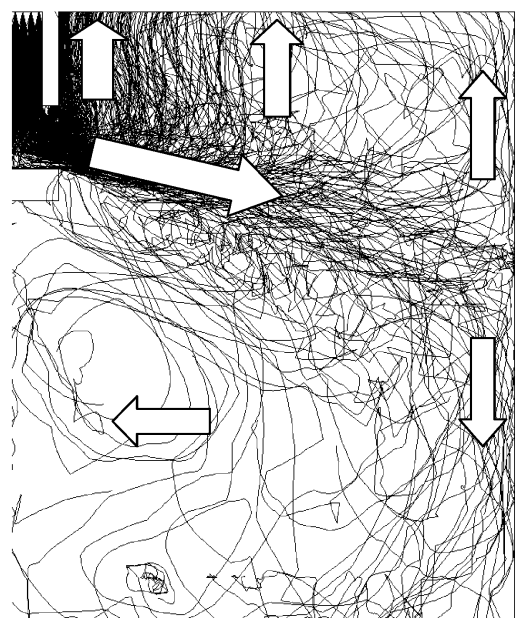

(c) $0.096 \mathrm{~T}$

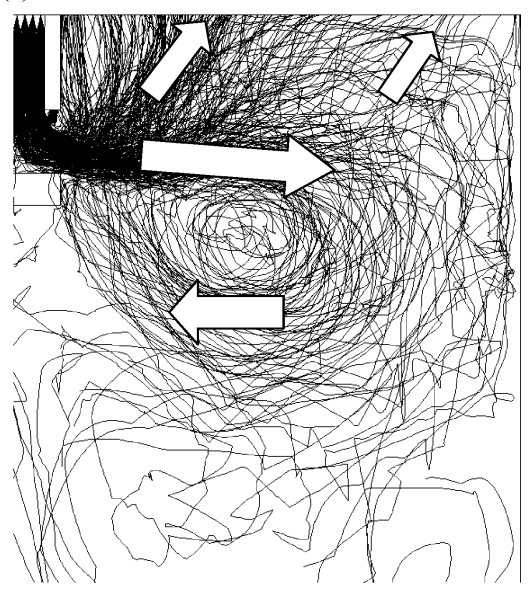

Fig. 13. Argon gas trajectories. (a) $0 \mathrm{~T}$, (b) $0.072 \mathrm{~T}$, (c) $0.096 \mathrm{~T}$. 


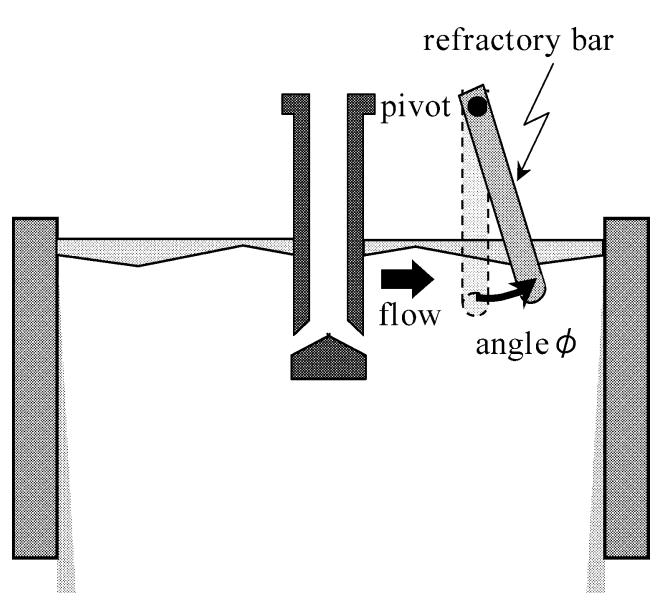

Fig. 14. Schematic view of surface velocity measurement apparatus.

cend up to the free surface as soon as they are supplied from the port of the nozzle. Smaller argon gas bubbles go to the narrow face wall. Then, some ascend up to the free surface, and some are pushed down to the inside of the mold. The argon gas distribution under 0.072 Tesla is similar to the case without a magnetic field, although some argon gas exists below the nozzle. In the case of 0.096 Tesla imposed case, argon gas bubbles rotate below the inlet flow where a strong magnetic force makes the circulation flow.

\subsection{Comparing Observation and Calculation}

The results of the simulation are validated by the velocity measurement of the molten steel and the investigation of the argon gas floating distribution in an actual operational continuous casting mold.

\subsubsection{Evaluation of Surface Velocity}

We measured the surface velocity using an immersed bar. ${ }^{12)}$ The schematic view of the surface velocity measurement apparatus is shown in Fig. 14. One edge of the immersion bar was supported. The angle $\phi$ of inclination of the bar by the molten steel flow was measured. From the moment of balance around the pivot of the bar, the angle $\phi$ is converted to the velocity magnitude. The measurement was performed at the $1 / 4$ mold width for several different imposed magnetic field intensity.

A comparison of the measured results and the calculated results is shown in Fig. 15. As a reference, the simulation data in which the argon gas is not supplied is also plotted. The surface velocity at the vertical axis is normalized by the value at 0 Tesla in order to evaluate the braking ratio. Positive velocity indicates flow in a direction toward the nozzle and vice versa. In the case of no argon gas bubbling, when the surface velocity is positive, the simulation result is in relatively good agreement with the measured one. After the surface velocity changes to zero increasing the imposed magnetic field, in the numerical simulation the surface velocity changes its direction slowly, whereas in the actual case the surface velocity changes its direction rapidly. On the other hand, when the effect of the molten steel jet dispersion due to the argon gas floatation is taken into considered in the simulation, good accuracy is obtained even after the surface velocity shifts to the minus flow direction.

As we have seen in Fig. 8, the calculated surface velocity

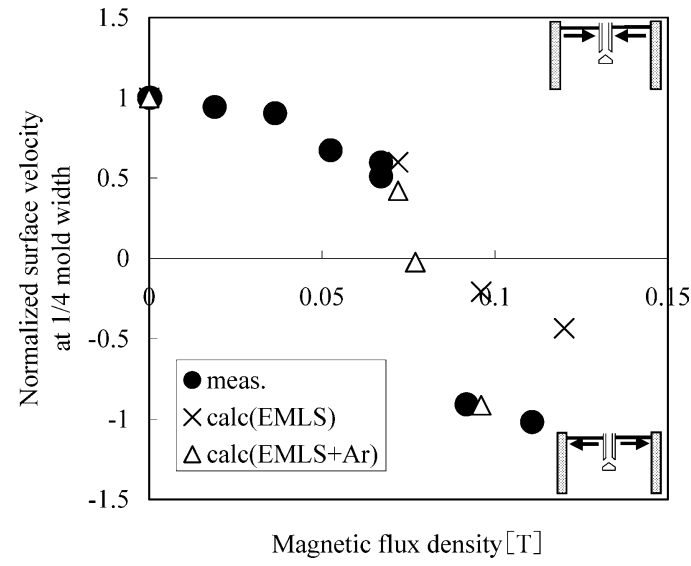

Fig. 15. Change in surface velocity at $1 / 4$ mold width with magnetic flux density.

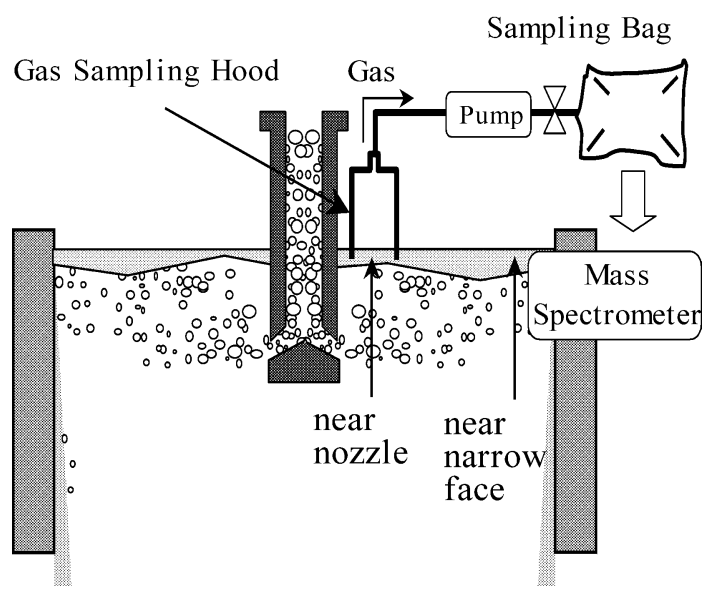

Fig. 16. Schematic view of argon gas detecting apparatus.

fits the measured data when the argon gas is supplied but the magnetic field is not imposed. Therefore, when the braking ratio is the same as each other, it means that the absolute value of the velocity is also the same. On the other hand, the calculated velocity without argon gas bubbling is higher than the measured one as shown in Fig. 8. In this case, it should be noted that even when the braking ratio of the calculated surface velocity is in good agreement with the measured one, the absolute velocity value is different between each other. It is obvious that the argon gas bubbling has to be considered if the absolute velocity value would like to be discussed.

\subsubsection{Argon Gas Floating Distribution}

The schematic view of the argon gas detecting apparatus $^{32)}$ is shown in Fig. 16. A sampling hood was placed over the free surface, and the floating gas was pumped up from the hood into a sampling bag. The gas sample was analyzed by a mass spectrometer, and the argon gas floating distribution was calculated. Sampling was performed near the nozzle and the narrow face of the mold.

Experimental data was set according to the argon gas volume fraction at the nozzle inlet. The argon gas floating ratio near the nozzle to the total is plotted in Fig. 17. "The total" means the sum of the argon gas floating amount near the nozzle and near the narrow face. As the injected argon gas volume fraction is increased, the argon gas floating ratio near the nozzle is increased, because larger argon gas bub- 


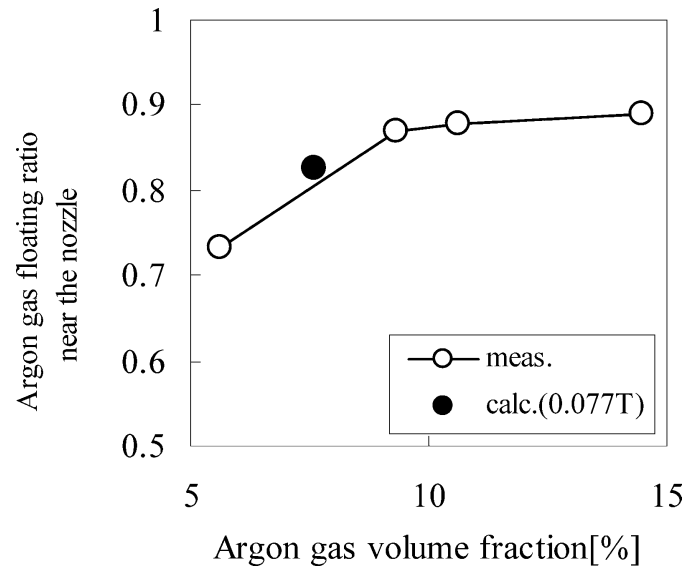

Fig. 17. Argon gas floating ratio near the nozzle.

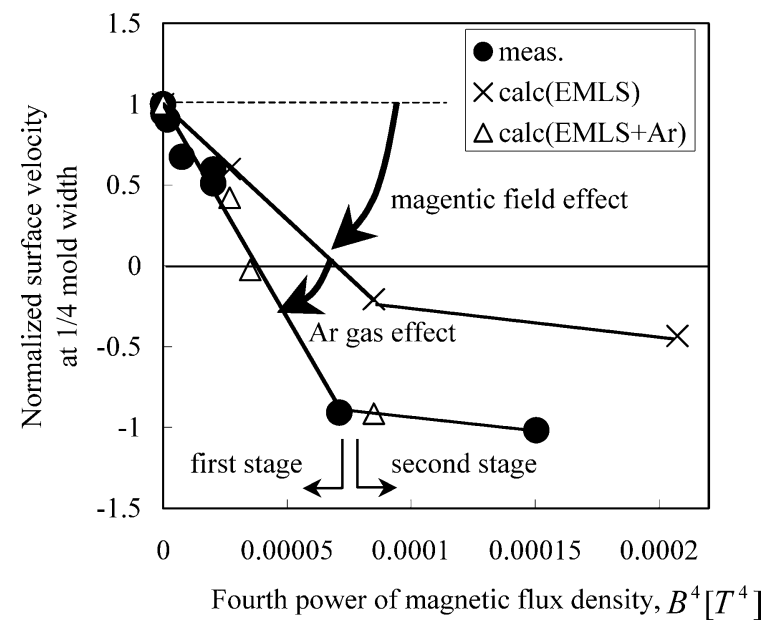

Fig. 18. Change in surface velocity at $1 / 4$ mold width with the fourth power of magnetic flux density.

bles are formed with an increase in the argon gas flow rate.

The calculated argon gas floating ratio near the nozzle to the total is also plotted in Fig. 17. The 0.077 Tesla imposed magnetic field case corresponds to the actual casting condition. The calculated data agrees with the measured curve. It can be said that this numerical calculation method can be applied to simulate the flow under a magnetic field with argon gas bubbling.

\subsection{Braking Effect of Magnetic Field and Argon Gas to the Surface Flow}

J. Kubota et al. ${ }^{12,37)}$ indicates that when the EMLS system is applied, the electromagnetic braking ratio is proportional to the fourth power of the imposed magnetic field, $B^{4}$. Therefore, Fig. 15 is redrawn using $B^{4}$ and shown in Fig. 18. As is expected, the calculated data under the magnetic field with argon gas bubbling fits the measured surface velocity profile. In addition, these data are reduced in proportion to $B^{4}$ at the "first stage". Calculated data without argon gas bubbling is also proportional to $B^{4}$ at the "first stage", although the braking ratio to the surface velocity is smaller than that of the argon gas bubbling case. This braking ratio without argon gas bubbling just shows the electromagnetic effect. From Fig. 18, it can be seen that argon gas bubbling contributes to the increase in the braking ratio, and its effect is considerably large. Argon gas makes the upstream of the molten steel near the nozzle, and it flows towards the narrow face of the mold near the free surface. This argon gas induced flow helps the magnetic field braking effect. It is found that the argon gas effect should be considered to evaluate the braking effect accurately.

At the "second stage", the surface velocity change becomes moderate. Once the molten steel starts to flow toward the mold center in the imposed magnetic field area, and after it makes a circulation in the lower part of the mold composed of the main flow from the nozzle to the narrow face of the mold, downstream near the wall and again induced flow from the narrow face of the mold to the mold center (see Fig. 11(b)), the molten steel flow near the free surface does not become much stronger. In addition, the electromagnetic force of the EMLS system is brought mainly from the moving velocity of the magnetic pole, as is discussed in Eqs. (1)-(3). To be precise, Eq. (1) should be expressed with the relative velocity between the moving velocity of the magnetic pole and the molten steel velocity where the magnetic field is imposed. In the EMLS system, as the magnetic pole moves from the narrow face of the mold to the mold center, the main electromagnetic force is in the same direction. The electromagnetic force becomes stronger, the molten steel starts to flow toward the mold center in the imposed magnetic field area, and the relative velocity between the pole moving velocity and induced molten steel flow velocity becomes smaller. Consequently, the effect of electromagnetic force increases no more.

\section{Conclusion}

The molten steel flow in a mold was computed when the magnetic field was imposed with the argon gas bubbling. From the results, the following conclusions were obtained.

(1) The EMLS system can change the surface flow magnitude and the direction by adjusting the intensity of the magnetic field. Argon gas has an effect to support the surface flow toward the narrow face of the mold.

(2) Increasing the imposed magnetic field, the braking ratio of the magnetic field is proportional to the fourth power of the magnetic field, $B^{4}$ at the "first stage" in both argon gas injected and non-injected case. Due to the argon gas supporting, the braking ratio in argon gas bubbling case becomes much larger than that in non-argon gas bubbling case. Argon gas bubbling cannot be neglected to evaluate the braking effect of the EMLS system.

(3) When compared with the observation of the practical casting, the numerical simulation method discussed in this paper is found to be applicable to evaluate the surface velocity and the argon gas floating distribution. By means of calculation, it can be said that an optimum intensity of a magnetic field for the flow control should be chosen in consideration with argon gas bubbling.

\section{REFERENCES}

1) Y. Yamaoka, M. Suzuki and K. Murakami: CAMP-ISIJ, 6 (1993), 279.

2) H. Tanaka, H. Kuwatori and R. Nishihara: Tetsu-to-Hagané, 78 (1992), 761.

3) N. Kasai, M. Kawasaki, K. Hanazaki and T. Sakashita: CAMP-ISIJ, 3 (1990), 1114

4) M. Iguchi, J. Yoshida, T. Shimizu and Y. Mizuno: ISIJ Int., 40 
(2000), 685.

5) Y. Otsuka, H. Yuyama, S. Kashio and S. Suzuki: CAMP-ISIJ, 3 (1990), 1225

6) T. Katai, Y. Otsuka, K. Shigematsu, T. Inomoto and H. Iiboshi: CAMP-ISIJ, 11 (1998), 861.

7) T. Yamazaki, H. Hasegawa, T. Toh and T. Inomoto: CAMP-ISIJ, 10 (1997), 236.

8) H. Tozawa, S. Idogawa, H. Nakato and K. Sorimachi: CAMP-ISIJ, 9 (1996), 604.

9) R. Nishimachi, J. Kubota, Y. Ogura, T. Ishii, M. Suzuki and M. Nakada: CAMP-ISIJ, 8 (1995), 952.

10) T. Teshima, J. Kubota, M. Suzuki, K. Ozawa, T. Masaoka and S. Miyahara: Tetsu-to-Hagané, 79 (1993), 576.

11) J. Kubota, S. Kuriyama and T. Teshima: CAMP-ISIJ, 2 (1989), 301.

12) J. Kubota, N. Kubo, M. Suzuki, T. Ishii, R. Nishimachi and N Aramaki: Tetsu-to-Hagané, 86 (2000), 271.

13) K. Takatani: CAMP-ISIJ, 16 (2003), 21

14) Z. Qian, Y. Wu, B. Li and J. He: ISIJ Int., 42 (2002), 1259.

15) T. Mochida, Y. Kishimoto, T. Yamada, H. Iijima, S. Nara and S Takeuchi: Tetsu-to-Hagané, 88 (2002), 393.

16) K. Okazawa, T. Toh, J. Fukuda, T. Kawase and M. Toki: ISIJ Int., 41 (2001), 851.

17) M. Morishita, M. Kogita, T. Nakaoka and T. Miyake: Tetsu-toHagané, 87 (2001), 167.

18) D. Kim, W. Kim and K. Cho: ISIJ Int., 40 (2000), 670

19) K. Okazawa, I. Sawada, H. Harada, T. Toh and E. Takeuchi: Tetsu-toHagané, 84 (1998), 490.

20) S. Nara, K. Sorimachi, N. Bessho, K. Kariya and R. Asaho: CAMPISIJ, 10 (1997), 764

21) H. Mizukami, S. Hiraki and T. Watanabe: Tetsu-to-Hagané, 86
(2000), 152.

22) M. B. Assar, P. H. Dauby and G. D. Lawson: Steelmaking Conf. Proc., ISS, Warrendale, PA, (2000), 397.

23) Z. Wang, K. Mukai and D. Izu: ISIJ Int., 39 (1999), 154

24) K. Takatani, Y. Tanizawa, H. Mizukami and K. Nishimura: ISIJ Int., 41 (2001), 1252.

25) T. Ishii, N. Kubo, M. Suzuki, M. Nakada, J. Kubota and R. Nishimachi: CAMP-ISIJ, 9 (1996), 212.

26) B. G. Thomas, X. Huang and R. C. Sussman: Metall. Mater. Trans. B., 25B (1994), 527.

27) B. G. Thomas and L. Zhang: ISIJ Int., 41 (2001), 1181.

28) T. Toh, H. Hasegawa and H. Harada: ISIJ Int., 41 (2001), 1245.

29) B. Li, T. Okane and T. Umeda: Metall. Mater. Trans. B., 31B (2000), 1491.

30) B. E. Launder and D. B. Spalding: Comp. Meth. Appl. Mech. Eng., 3 (1974), 269.

31) Fluent 5 User's Guide, Fluent Inc., Lebanon, NH, (1998).

32) N. Kubo, T. Ishii, J. Kubota and N. Aramaki: ISIJ Int., 42 (2002), 1251.

33) S. T. Johansen, F. Boysan and W. H. Ayers: Appl. Sci. Res., 44 (1987), 197.

34) S. S. Sazhin, M. Makhlouf and T. Ishii: Int. J. Numer. Meth. Fluids, 21 (1995), 433

35) T. Ishii , S. S. Sazhin and M. Makhlouf: Ironmaking Steelmaking, 23 (1996), 267.

36) H. Turkoglu and B. Farouk: Proc. ASME Winter Annual Meeting, Industrial Application of Fluid Mech., ASME, New York, (1990), 31 .

37) J. Kubota, N. Kubo, T. Ishii, M. Suzuki, N. Aramaki and R. Nishimachi: NKK Tech. Rev., 85 (2001), 1. 